\title{
Local contraction analysis of stochastic systems with limit
} cycles

\section{Conference Paper}

Author(s):

Ahbe, Eva; lannelli, Andrea (D); Smith, Roy (D)

Publication date:

2020

Permanent link:

https://doi.org/10.3929/ethz-b-000461411

Rights / license:

In Copyright - Non-Commercial Use Permitted

Originally published in:

https://doi.org/10.1109/CDC42340.2020.9304136

Funding acknowledgement:

178890 - Modeling, Identification and Control of Periodic Systems in Energy Applications (SNF) 


\title{
Local contraction analysis of stochastic systems with limit cycles
}

\author{
Eva Ahbe Andrea Iannelli Roy S. Smith \\ presented at: 2020 IEEE Conference on Decision and Control (2020), \\ DOI: $10.1109 /$ CDC 42340.2020 .9304136 , (02020 IEEE.
}

\begin{abstract}
A method is presented to obtain inner estimates of the region of transverse contraction (ROTC) which are invariant regions in which trajectories of a stochastic system converge to a stochastic limit cycle. Using the framework of Polynomial Chaos Expansions (PCE) the stochastic system is represented by a higher dimensional deterministic system. First, the connection between the stability of the periodic orbits of the stochastic system and the stability of the limit cycle of its PCE system is established. Then transverse contraction criteria, as well as invariance conditions, are formulated for the PCE system to certify an ROTC estimate for the PCE system. From this, and by leveraging the established stability connection, an ROTC estimate of the stochastic system is retrieved. Finally, an optimization program, based on matrix sum-of-squares verification techniques, to implement the contraction and invariance criteria is proposed.
\end{abstract}

\section{INTRODUCTION}

The analysis of regions in which a limit cycle is a stable attractor is of relevance to many applications, such as robotics [1], biology [2], aerospace [3] and wind energy [4]. While for deterministic systems these regions have been widely studied, attractive regions of limit cycles of uncertain systems, which represent a broader and more realistic class of systems, have been much less considered. In [5] robust sets in a state-action space are obtained from viability theory for limit cycles of systems representing legged locomotion. In [6] two methods based on describing functions and integral quadratic constraints are proposed to analyze the stability of limit cycles of an aeroelastic plant. One of the reasons contributing to the relatively sparse coverage of this research is that, in general, the location of the periodic orbit of an uncertain system depends on the realization of the uncertainty. This excludes the use of well-established Lyapunov arguments for the analysis of the attractive region as these require the knowledge of the location of the attractor.

An approach for stability analysis which does not require the information on the limit cycle location is given by contraction methods [7]. In contraction analysis the evolution of an incremental distance defined between any two neighboring trajectories in a given region is considered. If the distance is decreasing over time then all trajectories in the region eventually converge to a single trajectory. Since two neighboring trajectories which converge to a periodic orbit

This work was partially supported by the Swiss National Science Foundation under grant no.: 200021_178890.

The authors are with the Automatic Control Laboratory, Swiss Federal Institute of Technology (ETH Zurich), Physikstrasse 3, 8092 Zurich, Switzerland, \{ahbee,iannelli,rsmith\}@control.ee.ethz.ch. will never converge to a single trajectory, a weaker form of contraction for systems with periodic solutions is considered. This is called transverse contraction and requires contraction to hold only in the subspace which is transversal to the system's flow [8], [9]. While transverse contraction has been studied for deterministic systems [9], [8], the application to uncertain systems has so far mostly been limited to the case of affine uncertainty [4].

Many analysis methods for the stability of limit cycles are based on Poincaré maps or suitable modifications of it [1], [4]. While being a powerful tool, they are often only welldefined in a region close to the limit cycle and significantly increase the computationally complexity of the problem.

In this work, we propose an approach to inner approximate the attracting region of a limit cycle, which we refer to as region of transverse contraction (ROTC). Our approach extends the existing methods in the following ways.

We provide an analysis tool which is applicable to the broad class of stochastic systems given by second order random processes. These systems have finite second moment and are affected by uncertainty which can come from any probability distribution with finite second moment. As such, second order processes represent most processes of the real world [10]. By using the framework of Polynomial Chaos Expansion (PCE), second order processes can be represented by a higher dimensional deterministic system. For an overview of PCE see, e.g., [11]. By applying PCE to stochastic systems with limit cycles we allow to consider uncertainties coming from a wide range of probability distributions as opposed to most existing approaches which employ robust control-based deterministic approaches. With a similar motivation, PCE has been previously employed to analyse regions of attraction of stochastic systems with equilibrium points in [12]. With respect to limit cycles, PCE has been used to investigate the statistical properties of stochastic periodic orbits [13], [14]. While in [13] the authors have found PCE to accurately represent the spatial statistics of stochastic systems with limit cycles for short time horizons, the limitations of PCE for analysing the purely spatial statistics of limit cycles such as peak amplitude variations are revealed. These limitations are due to the nature of PCE which represents combined spatial and temporal statistics. In this work we explore these limitations and leverage the accuracy of PCE in representing the combined statistics of limit cycles. Based on these finding a connection between the periodic behavior of both the stochastic and the PCE 
system representations is drawn. This allows the analysis of the attractive regions of the stochastic system's limit cycle by considering the deterministic PCE representation. Based on the PCE representation we further extend the existing transverse contraction notions by formulating rigorous criteria for an invariant and contracting region around the stochastic limit cycle which provide an inner estimate of the ROTC. An optimization program is proposed which employs sumof-squares verification tools for polynomial matrices [15], [4] to compute an inner estimate of the ROTC. The method is demonstrated by an example from the literature.

\section{A. Notation}

Let $(\Theta, \mathcal{F}, \mu)$ be a probability space, where $\Theta$ is a sample space, $\mathcal{F}$ is a $\sigma$-algebra and $\mu$ is the probability measure. A random variable with finite second moment is denoted by $\xi: \Theta \rightarrow \mathbb{R}, \xi \in \mathcal{L}_{2}(\Theta, \mu)$ where $\mathcal{L}_{l}, 1 \leq l \leq \infty$, is the Lebesgue space (see, e.g. [11]). Here, $\xi$ is considered onedimensional for simplicity, although the proposed approach also holds for multidimensional random variables. Let $\mathcal{P}^{d}$ denote the ring of all $d$-variate polynomials with real coefficients and let $\mathcal{P}_{\leq r}^{d}$ denote those polynomials of total degree at most $r \in \mathbb{N}_{0}$. A polynomial $g(x): \mathbb{R}^{n} \rightarrow \mathbb{R}, g(x) \in \mathcal{P}_{\leq r}^{d}$ is called a sum-of-squares (SOS) if it can be written as $g(x)=$ $\sum_{i} q_{i}(x)^{2}, q_{i}(x) \in \mathcal{P}_{\leq r / 2}^{n}$. Moreover, $g$ is SOS if and only if there is a matrix $Q \succeq 0$ such that $g(x)=v(x)^{T} Q v(x)$, where $v(x)$ is the monomial vector. The set of all SOS polynomials in the indeterminant $x$ is indicated by $\Sigma[x]$. The polynomial degree of $g$ is denoted by $\partial(g)$.

\section{Problem statement}

The aim of this work is to obtain an invariant region in which a stochastic system is transversely contracting to an uncertainty-dependent periodic orbit.

\section{A. Stochastic system and orbit}

We consider continuous time second order processes,

$$
\dot{x}(t, \xi)=f(x(t, \xi), \phi(\xi)),
$$

where $x \in \mathbb{R}^{n}$ is the random state variable, $\phi(\xi) \in$ $\mathcal{L}_{2}\left(\Theta, \mu ; \mathbb{R}^{m}\right)$ is a vector of $\mathcal{L}_{2}$-bounded uncertain parameters and $f: \mathbb{R}^{n} \times \mathbb{R}^{m} \rightarrow \mathbb{R}^{n}$ is a polynomial function in $x$ and $\phi$. The initial state is random as well, $x(0)=x_{\text {ini }}(\xi)$. We further assume the support of $\xi$ to be finite. Uncertainty distributions with typically infinite support, such as Gaussian distributions, can be considered by truncating the distribution tails, which in practice leads to negligible approximation errors [16]. In this work we consider stochastic systems (1) which, for initial conditions in some region $K \subset \mathbb{R}^{n}$, converge to an uncertainty-dependent periodic orbit for every realization of the random variable $\xi$.

Definition 1: Let $\psi\left(x_{\text {ini }}^{\dagger}, \xi^{\dagger}, t\right)$ denote the flow of the system (1) with initial condition $x_{\text {ini }}^{\dagger}$ and for a realization $\xi^{\dagger} \in \Theta$. A periodic orbit $\Gamma_{\xi^{\dagger}}$ is defined by a solution $u\left(t, \xi^{\dagger}\right)=\psi\left(x_{\text {ini }}^{\dagger}, \xi^{\dagger}, t\right)$ which satisfies $u\left(0, \xi^{\dagger}\right)=u\left(T_{\xi^{\dagger}}, \xi^{\dagger}\right)$ with the period given by the minimum nontrivial $T_{\xi^{\dagger}}>0$,

$$
\Gamma_{\xi^{\dagger}}=\left\{x \in \mathbb{R}^{n} \mid x=u\left(t, \xi^{\dagger}\right), t \in\left[0, T_{\xi^{\dagger}}\right]\right\} .
$$

In particular, the periodic solution $u(t, \xi)$ is considered to depend continuously on the random variable. The union of periodic orbits for all realizations of $\xi \in \Theta$ is denoted by

$$
\Gamma:=\bigcup_{\xi \in \Theta} \Gamma_{\xi},
$$

and refered to as the stochastic periodic orbit of (1).

\section{B. Local stability of periodic orbits}

Asymptotic stability of an equilibrium point is concerned with the convergence of trajectories of a system to a point in the state space. When convergence of trajectories to a limit cycle is considered a different notion of asymptotic stability is needed as two neighboring trajectories do not converge to a single point but instead to a set in which they will remain apart. Thus, the notion of asymptotic orbital stability can be defined instead [17]. The definition for a deterministic system $f_{\text {det }}(x)$ with periodic orbit $\Gamma_{\text {det }}$ is given as follows.

Definition 2: Let $K \subseteq \mathbb{R}^{n}$ be a region with $\Gamma_{\text {det }} \subset K$. The periodic orbit $\Gamma_{\text {det }}$ is called asymptotically orbitally stable if it is stable and attractive. It is stable if $\forall \epsilon>0$, there is a $\delta>0$ such that $\forall x_{\text {ini }} \in K$ with $\operatorname{dist}\left(x_{\text {ini }}, \Gamma_{\text {det }}\right)<\delta$ we have that $\operatorname{dist}\left(\psi\left(x_{\text {ini }}, t\right), \Gamma_{\text {det }}\right)<\epsilon, \forall t>0$. It is further attractive if there is a $\delta>0$ such that $\forall x_{\text {ini }} \in K$ with $\operatorname{dist}\left(x_{\text {ini }}, \Gamma_{\text {det }}\right)<\delta$ we have $\lim _{t \rightarrow \infty} \operatorname{dist}\left(\psi\left(x_{\text {ini }}, t\right), \Gamma_{\text {det }}\right)=0$.

We extend this definition for a stochastic system (1).

Definition 3: The stochastic periodic orbit $\Gamma$ as given in (3) is called stochastically asymptotically orbitally stable if Definition 2 holds for the periodic orbits $\Gamma_{\xi}$ of all $\xi \in \Theta$. That is, $\exists \delta$ such that $\forall x_{\text {ini }} \in K$ with $\operatorname{dist}\left(x_{\text {ini }}, \Gamma\right)<\delta$ we have $\lim _{t \rightarrow \infty} \operatorname{dist}\left(\psi\left(x_{\text {ini }}, \xi, t\right), \Gamma\right)=0, \forall \xi \in \Theta$.

With Definition 3, we introduce the set $\mathcal{R}^{*}$

$$
\mathcal{R}^{*}:=\left\{x_{\mathrm{ini}} \in \mathbb{R}^{n} \mid \lim _{t \rightarrow \infty} \operatorname{dist}\left(\psi\left(x_{\mathrm{ini}}, \xi, t\right), \Gamma\right)=0, \forall \xi \in \Theta\right\},
$$

which is the region in which a system is converging to its stochastic limit cycle $\Gamma$. Often referred to as region of attraction in studies of attractive equilibrium points, for reasons presented in the following we refer to $\mathcal{R}^{*}$ as the region of transverse contraction (ROTC) of the system (1).

\section{Polynomial Chaos Expansion}

Polynomial Chaos Expansions (PCE) can be used to approximate stochastic processes with finite second moment. The PCE of a stochastic system results in a deterministic representation of the system at the cost of a higher state dimension. For an overview see, e.g., [11].

To approximate random variables with PCE, an orthogonal polynomial basis $\mathcal{Q}=\left\{\Phi_{i} \mid i \in \mathbb{N}\right\} \subseteq \mathcal{P}$ is defined using

$$
\left\langle\Phi_{i}(\xi), \Phi_{j}(\xi)\right\rangle=\int_{\Theta} \Phi_{i}(\xi) \Phi_{j}(\xi) d \mu(\xi)=\gamma_{i} \delta_{i j}
$$

where $\gamma_{i}:=\left\langle\Phi_{i}(\xi), \Phi_{i}(\xi)\right\rangle$ is the normalization factor and $\langle\cdot, \cdot\rangle$ denotes the inner product in $\mathcal{L}_{2}$, representing integration (i.e. expectation) with respect to $\mu$. For optimal convergence of the expansion, there is a specific orthogonal polynomial basis corresponding to the type of probability distribution of 
the random variable [10]. The PCE of a square-integrable vector-valued random variable $y(\xi) \in \mathcal{L}_{2}\left(\Theta, \mu ; \mathbb{R}^{n}\right)$ is then

$$
y(\xi)=\sum_{i=0}^{\infty} \bar{y}_{i} \Phi_{i}(\xi)
$$

with vector valued PCE coefficients, also referred to as the stochastic modes, $\bar{y}_{i}=\left[\bar{y}_{1_{i}}, \ldots, \bar{y}_{n_{i}}\right]^{T}$, which are obtained, e.g., from a Galerkin projection

$$
\bar{y}_{i}=\gamma_{i}^{-1}\left\langle y(\xi), \Phi_{i}(\xi)\right\rangle .
$$

The expansion series in (6) is infinite and needs to be truncated for practical purposes. With a truncation order $p$, the PCE represents a $n$-dimensional stochastic system as a $n(p+1)$-dimensional deterministic system. The deterministic equations are obtained by projecting the system onto each of the $(p+1)$ basis functions, as in (7). See e.g. [12] for details. We denote all PCE coefficients of $y(\xi)$ by $\bar{y}=\left[\bar{y}_{0}, \ldots, \bar{y}_{p}\right]^{T}$, with each stochastic mode $\bar{y}_{i}$ as defined above.

The moments of the random variable $y(\xi)$ can be retrieved from the PCE coefficients of its $\mathcal{L}_{2}$-optimal expansion. The first moment (mean) of $y(\xi)$ is found as

$$
m:=\mathbb{E}[y(\xi)]=\left\langle y(\xi), \Phi_{0}\right\rangle=\bar{y}_{0} .
$$

The second central moment (variance) of $y(\xi)$ is given by

$$
\sigma^{2}:=\mathbb{E}\left[|y(\xi)-\mathbb{E}[y(\xi)]|^{2}\right]=\sum_{j=1}^{p} \bar{y}_{j}^{2} \gamma_{j},
$$

where the sum is to be taken separately over all PCE coefficients of each component of $y$.

The PCE of a stochastic system (1) is denoted by

$$
\dot{\bar{x}}=\bar{f}(\bar{x}),
$$

where $\bar{x} \in \mathbb{R}^{n(p+1)}$ are the vector of PCE coefficients of the state $x \in \mathbb{R}^{n}$, and $\bar{f}: \mathbb{R}^{n(p+1)} \rightarrow \mathbb{R}^{n(p+1)}$ specifies the PCE coefficient dynamics. The overbar notation indicates variables in the PCE representation.

\section{PCE for limit cycle systems}

It has been previously shown [18] that PCE's of stochastic systems with limit cycles are able to accurately represent the short-time statistical variations of the system's trajectories. However, the PCE fails to accurately reproduce the spatial statistical properties (such as the location of the mean limit cycle, or the variance of particular solutions of interests such as peak amplitude values [3]) of the periodic solution for long time spans, even for high truncation orders. Several modified PCE approaches have been proposed to obtain more accurate long-term representations of the purely spatial variations of a limit cycle. For example, an equation-free approach essentially resulting in studying the dynamics on Poincarémap-like hyperplanes was proposed in [19]. Alternatively, a better suited basis for oscillatory responses can be used, such as Wiener-Haar wavelets proposed in [18], or Fourier polynomials which were introduced in [3].

For the purpose of the analysis presented here, the exact spatial statistics of the periodic orbit are not required to be known. This allows us to leverage short-time accuracy of the PCE in order to represent the statistical behavior of the trajectories in the vicinity of a periodic solution. As for the periodic solution itself we take advantage of the fact that the qualitative behavior is still accurately captured by the PCE. That is, the PCE dynamics are able to discriminate between the existence or not of a periodic attractor, while we do not attempt to use it to characterize other properties, e.g., the mean or variance of amplitude and/or frequency. More precisely, we find the following.

Lemma 1: If the system (1) has a stochastic periodic solution $u(t, \xi)$, then the PCE coefficient system (10) also has a periodic solution $\bar{u}(t)$.

Proof: The PCE coefficients of a random process are obtained from the projection (7), in particular

$$
\begin{aligned}
\bar{f}(\bar{x}(t)) & =\gamma^{-1}\left\langle f(x(t, \xi), \phi(\xi)), \Phi_{j}(\xi)\right\rangle \\
& =\gamma^{-1} \int_{\Theta} f(x(t, \xi), \phi(\xi)) \Phi_{i}(\xi) d \mu(\xi) .
\end{aligned}
$$

Since the polynomial basis $\left\{\Phi_{i}(\xi)\right\}_{i=0}^{\infty}$ is time invariant, the projection of a variable or process which is periodic for all $\xi$ in the domain of integration onto a member $\Phi_{i}(\xi)$ of the basis is a linear combination of periodic quantities and thus is also periodic.

Similarly as before for the stochastic system, we define for the PCE system the set containing the periodic solution as

$$
\bar{\Gamma}=\left\{\bar{x} \in \mathbb{R}^{n(p+1)} \mid \bar{x}=\bar{u}(t), t \in[0, T]\right\} .
$$

For small $t$, the periodic solution of (10) represents the spatial statistics of the periodic solutions $u(t, \xi)$ but for $t \gg 0$ the PCE solutions converge to a periodic behavior which differs from the spatial statistics of $u(t, \xi)$. This can be intuitively understood by considering that PCE represents the 'full' statistics, which includes spatial and temporal statistics. Trajectories starting from the same initial condition in the set $\Gamma$ but with a different realization of the uncertainty will, in general, not only vary in the exact location of the periodic solution but also in the speed with which they travel the orbit, i.e. their frequency. Considering, for example, the mean of the spatial variability over all trajectories after a certain time $t$ has passed, one clearly sees that, in general, they differ significantly from the spatial variability one would expect to find from, e.g., hyperplane considerations. An illustration of the temporal and spatial variation is provided in Figure 1. Note, that the reverse of Lemma 1 does not necessarily hold when the expansion (6) is truncated. Since we consider second order processes the expansion series is, however, convergent and in the remainder we will work under the commonly made assumption that the truncated PCE system represents the properties under consideration (i.e., short-time accuracy of statistics and periodicity of solutions) accurately. To consider the truncation error, procedures as in [20] and [21] can be taken into account.

\section{REgions of TRANSVERSE CONTRACTION}

The aim in this work is to obtain inner estimates of $\mathcal{R}^{*}$ by means of PCE representations. The first step towards 


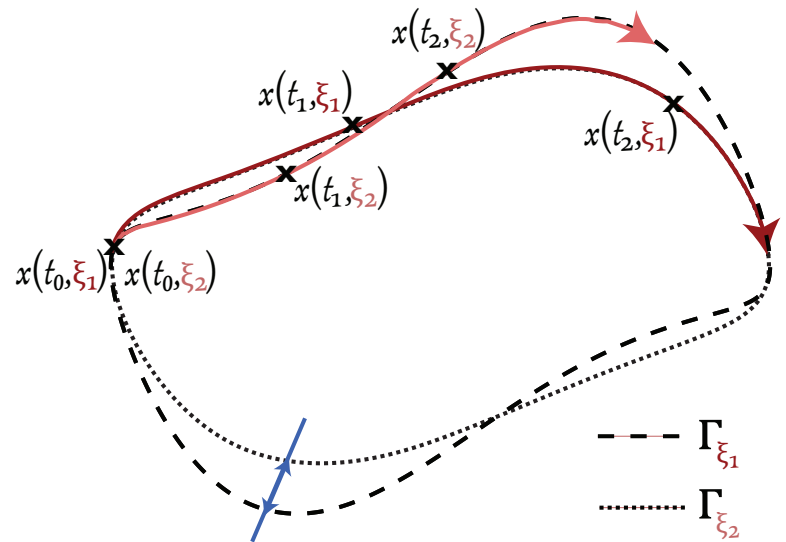

Fig. 1. Illustration of two trajectories starting from the same initial point for two different realizations of the uncertainty, $\xi_{1}$ and $\xi_{2}$. Both follow a limit cycle corresponding to their uncertainty. The state of both trajectories at the same time $t_{1}$ and $t_{2}$ is indicated. The mean and variance of those states for each fixed time (as given by the PCE representation) clearly differ from a purely spatial mean and variance at a fixed location, here indicated by the hyperplane in blue.

this goal is to establish a connection between the stochastic orbital stability of a the stochastic orbit and the deterministic orbital stability of the PCE orbit.

Proposition 1: Let $K \subset \mathbb{R}^{n}$ be a region such that $\Gamma \subset K$ and further $\bar{\Gamma} \subset \bar{K} \subset \mathbb{R}^{n(p+1)}$, where

$$
\bar{K}=\left\{\bar{x} \in \mathbb{R}^{n(p+1)} \mid \bar{x}_{i}=\gamma_{i}^{-1}\left\langle x, \Phi_{i}\right\rangle, x \in K\right\} .
$$

Then the orbit $\Gamma$ defined in (3) is stochastically asymptotically orbitally stable in $K$ if the PCE orbit $\bar{\Gamma}$ is asymptotically orbitally stable in the region $\bar{K}$.

Proof: Asymptotic orbital stability of $\bar{\Gamma}$ in $\bar{K}$ implies that any solution starting at $\bar{x}_{\text {ini }}$ in the neighborhood of $\bar{\Gamma}$ eventually converges to it, i.e. $\bar{\psi}\left(\bar{x}_{\text {ini }}\right) \rightarrow \bar{\Gamma}$ as $t \rightarrow \infty$. Further, $x_{\text {ini }}(\xi)=\sum_{i=0}^{p} \bar{x}_{\text {ini } i} \Phi_{i}(\xi)$ from the PCE representation (6). Let $\bar{\psi}(\cdot, \cdot)$ be the flow of the PCE system (10). Assume, that $\exists x_{\text {ini }}(\xi)$ for which $\psi\left(x_{\text {ini }}(\xi), t\right) \not \rightarrow \Gamma$ as $t \rightarrow \infty$. Then $\lim _{t \rightarrow \infty} \psi\left(x_{\text {ini }}(\xi), t\right)=\lim _{t \rightarrow \infty} \sum_{i=0}^{p} \bar{\psi}\left(\bar{x}_{\text {ini }}, t\right)_{i} \Phi_{i}(\xi)=$ $\sum_{i=0}^{p} \bar{u}_{i}(t) \Phi_{i}(\xi)=u(\xi, t)$, which is in contradiction with the assumption. The statement of the proposition follows. $\square$

Leveraging Proposition 1, the stochastic orbital stability of the orbit $\Gamma$ can be investigated by analyzing the orbital stability of the PCE orbit. The problem is addressed by first finding regions in which a PCE system is converging to $\bar{\Gamma}$ and then retrieving from them a region in which the stochastic system converges to $\Gamma$. This results in inner estimates of $\mathcal{R}^{*}$.

\section{A. Transverse contraction criteria}

Since the location of both orbits are in general unknown, Lyapunov arguments of stability cannot be employed for the stability analysis. Instead, contraction methods [7] can be applied. More precisely, for systems with limit cycles so called transverse contraction methods are used as the property of contraction is required to hold only for a subspace transversal to the flow of the system [8], [9]. Since the PCE system (10) is a deterministic system, the deterministic approach is used to analyze the transverse contraction proposed in [8].

Let $\delta$ be a virtual displacement, i.e. an infinitesimal displacement of $\bar{x}$ at fixed time for which a Finsler metric function
$D(\bar{x}, \delta)=\delta^{T} M(\bar{x}, \delta) \delta$ measuring the squared distance with respect to the metric $M$ can be introduced. Further, let $\bar{K} \subset \mathbb{R}^{n(p+1)}$ be a region with $\bar{\Gamma} \subset \bar{K}$. A system $\bar{f}(\bar{x})$ is said to be transversely contracting in $\bar{K}$ if there exists a Finsler function $D(\bar{x}, \delta)$ satisfying

$$
\frac{\partial D}{\partial \bar{x}} \bar{f}(\bar{x})+\frac{\partial D}{\partial \delta} \frac{\partial \bar{f}(\bar{x})}{\partial \bar{x}} \delta \leq-\beta D(\bar{x}, \delta),
$$

for all $\delta \neq 0$ and $\bar{x} \in \bar{K}$, with a convergence rate $\beta>0$, such that the orthogonality condition

$$
\frac{\partial D}{\partial \delta} \bar{f}(\bar{x})=\delta^{T} M \bar{f}(\bar{x})=0,
$$

holds. Conditions (14) and (15) imply that the distance between any two trajectories in $\bar{K}$ is decreasing in the transverse direction, i.e. orthogonal to the system flow. For a compact and positively invariant region $\overline{\mathcal{R}}^{*} \subseteq \bar{K}$ with $\bar{\Gamma} \subset \overline{\mathcal{R}}^{*}$ it further holds that for every two solutions $\bar{x}_{(1)}$ and $\bar{x}_{(2)}$ with initial conditions in $\overline{\mathcal{R}}^{*}$ there exists a time reparametrization $\tau(t)$ such that $\bar{x}_{(1)}(t) \rightarrow \bar{x}_{(2)}(\tau(t))$ as $t \rightarrow \infty$ [8]. From this follows that trajectories starting from initial conditions $\bar{x}_{\text {ini }} \in \overline{\mathcal{R}}^{*}$ converge to the unique limit cycle inside $\overline{\mathcal{R}}^{*}$ with convergence rate $\beta$. Formally, the region $\overline{\mathcal{R}}^{*}$ is then defined as:

$$
\overline{\mathcal{R}}^{*}=\left\{\bar{x}_{\text {ini }} \in \mathbb{R}^{n(p+1)} \mid \lim _{t \rightarrow \infty} \operatorname{dist}\left(\bar{\psi}\left(\bar{x}_{\mathrm{ini}}, t\right), \bar{\Gamma}\right)=0\right\},
$$

where $\bar{\psi}(\cdot, \cdot)$ is the flow of the PCE system (10). In the following, we are interested in finding inner estimates $\overline{\mathcal{R}}$ of the region $\overline{\mathcal{R}}^{*}$ of the PCE system and refer to such as ROTC estimate. The following Theorem uses results from Theorem 3 in [8] and Theorem 1 in [22] to provide conditions for a region to be an inner estimate of the ROTC.

Theorem 1: Let $B: \mathbb{R}^{n(p+1)} \rightarrow \mathbb{R}$ be a continuously differentiable function and let $\overline{\mathcal{R}}=\left\{\bar{x} \in \mathbb{R}^{n(p+1)} \mid B(\bar{x}) \leq\right.$ $\rho$ \} where $\rho$ is a positive scalar such that $\bar{\Gamma} \subset \overline{\mathcal{R}}$. If

- there exists a metric function $D(\bar{x}, \delta)=\delta^{T} M(\bar{x}, \delta) \delta$, a convergence rate $\beta$ and a function $\lambda(\bar{x}) \geq 0$, such that

$$
\begin{aligned}
C(\bar{x}) & \frac{\partial \bar{f}(\bar{x})^{T}}{\partial \bar{x}}+\frac{\partial \bar{f}(\bar{x})}{\partial \bar{x}} C(\bar{x})-\dot{C}(\bar{x})+ \\
& +2 \beta C(\bar{x})-\lambda(\bar{x}) S(\bar{x}) \leq 0, \quad \forall \bar{x} \in \overline{\mathcal{R}},
\end{aligned}
$$

where $S(\bar{x}):=\bar{f}(\bar{x}) \bar{f}(\bar{x})^{T}, C(\bar{x}):=M(\bar{x})^{-1}$ and $\dot{C}(\bar{x})=\frac{d}{d t}\left(M^{-1}(\bar{x})\right)=-M^{-1}(\bar{x}) \dot{M}(\bar{x}) M^{-1}(\bar{x})$, and

- the derivative of $B$ with respect to time on the boundary of $\overline{\mathcal{R}}$ is negative, i.e.,

$$
\nabla B(\bar{x}) \bar{f}(\bar{x})<0, \forall \bar{x} \in \overline{\mathcal{R}}^{\circ},
$$

where $\overline{\mathcal{R}}^{\circ}:=\{\bar{x} \mid B(\bar{x})=\rho\}$,

then the region $\overline{\mathcal{R}}$ is transversely contracting and invariant. Thus, $\overline{\mathcal{R}} \subseteq \overline{\mathcal{R}}^{*}$, i.e., $\overline{\mathcal{R}}$ is an inner estimate of the ROTC of system (10), and all trajectories starting in $\overline{\mathcal{R}}$ converge to $\bar{\Gamma}$.

A proof of the first property can be found in [8], which shows how condition (17) certifies a positively invariant set to be a transversely contracting region of a deterministic system. The proof for condition (18) to show invariance of $\overline{\mathcal{R}}$ is presented, e.g., in [23] and is omitted here for brevity. 


\section{B. Retrieving $\mathcal{R}$ from $\overline{\mathcal{R}}$}

In order to retrieve an inner estimate $\mathcal{R}$ of the ROTC $\mathcal{R}^{*}$ of the stochastic system we use an approach similar to the one proposed in [12] for regions of attraction of equilibrium points. More precisely, with an ROTC estimate $\overline{\mathcal{R}}$ obtained for the PCE system from Theorem (1) we find the ROTC estimate $\mathcal{R}$ for the stochastic system from the following.

Lemma 2: Let $\overline{\mathcal{R}}$ be an inner estimate of the ROTC for the PCE system. Then the set

$$
\mathcal{R}=\left\{x_{\text {ini }} \mid x_{\text {ini }}(\xi)=\sum_{i=0}^{p} \bar{x}_{\text {ini } i} \Phi_{i}(\xi), \forall \bar{x}_{\text {ini }} \in \overline{\mathcal{R}}\right\}
$$

is an inner estimate of the ROTC for the stochastic system. This Lemma results from the PCE representation (6) and from Proposition 1 applied to the sets given by $\overline{\mathcal{R}}$ and $\mathcal{R}$.

The region $\mathcal{R}$ as given by equation (19) is a stochastic region. An explicit expression for this region can be obtained by fixing the variance of the initial states to a desired value, and representing the region $\mathcal{R}$ as the set of the mean values which are contained in $\overline{\mathcal{R}}$ with that fixed variance. For more details on this procedure we refer the reader to [12].

\section{Algorithm FOR COMPUTING ROTC}

In this section we propose an optimization program to compute an ROTC estimate $\overline{\mathcal{R}}$ for (1) by verifying the conditions of Theorem 1. The optimization program consists of two steps. In the first step, the region in which the system is contracting is maximized by testing condition (17). This contracting region will be denoted by $\overline{\mathcal{Z}}$. In the second step, the largest invariant set inside of the contracting region is extracted in order to obtain a transversely contracting and invariant region $\overline{\mathcal{R}}$.

\section{A. Step 1: Maximizing the contracting region}

In order to obtain a region $\overline{\mathcal{Z}}$ in which the system is transversely contracting a metric has to be found which satisfies condition (17). Let the region $\overline{\mathcal{Z}}$ be given by the sublevel set $\rho$ of a polynomial function $z(\bar{x})=v(\bar{x})^{T} Z v(\bar{x})$, i.e., $\overline{\mathcal{Z}}:=\{\bar{x} \mid z(\bar{x}) \leq \rho\}$, where $v(\bar{x})$ is the vector of monomials. The optimization program both aims at finding a contraction metric $M$ which satisfies the transverse contraction condition (17) for all $\bar{x} \in \overline{\mathcal{Z}}$, as well as maximize the region $\overline{\mathcal{Z}}$ itself. Since we consider polynomial systems in this work, the Positivstellensatz [24] can be used to obtain computationally verifiable expressions for constraints such as (17). The procedure consists of formulating set emptiness conditions which are then relaxed to sum-of-squares (SOS) programs. This approach was introduced in [24] and employed in several previous applications, e.g. in [25], [26] among others. Note that the contraction metric $M$ as well as the condition (17) are given by matrix polynomials (i.e. matrices with polynomial entries). Applying the Positivstellensatz procedure thus results in SOS matrix constraints. A matrix is SOS if it is symmetric, with polynomial entries $F(x) \in \mathbb{R}[x]^{n \times n}$, and if for a vector of new indeterminants $y=\left[y_{1}, \ldots, y_{n}\right]^{T}$ the scalar polynomial $y^{T} F(x) y$ is SOS in $\mathbb{R}[x, y]$ [15]. The set of SOS matrices in $x$ are in the following denoted by $\Sigma^{n \times n}[x]$.
In order to maximize the contracting region the program optimizes over the sublevel set size $\rho$, while at the same time optimizing over the shape of the region given by $Z$. To prevent an increase of $\rho$ by simple rescaling of $Z$ the trace of $Z$ is fixed to a constant value $a$. We then obtain the following optimization program.

$$
\begin{array}{rcc}
\max _{C(\bar{x}), \lambda(\bar{x}), s_{1}(\bar{x}), s_{2}(\bar{x}), Z} \rho & \\
\text { subject to } & C(\bar{x})-l & \in \Sigma^{n \times n}[\bar{x}], \\
-J(\bar{x})-\beta C(\bar{x})+\lambda(\bar{x}) S(\bar{x})- & \\
-s_{1}(\bar{x})(\rho-z(\bar{x}))-s_{2}(\bar{x})(b(\bar{x})-\epsilon) & \in \Sigma^{n \times n}[\bar{x}], \\
s_{1}(\bar{x}), s_{2}(\bar{x}) & \in \Sigma^{n \times n}[\bar{x}], \\
\lambda(\bar{x}) & \in \Sigma[\bar{x}], \\
Z \succ 0, \operatorname{tr}(Z)=a, &
\end{array}
$$

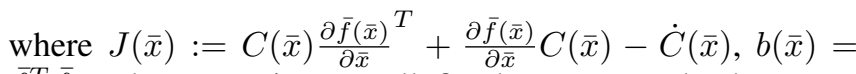
$\bar{f}^{T} \bar{f}$ and $\epsilon \ll 1$ is a small fixed constant. The last term in constraint (20c) serves to exclude equilibrium points at which the dynamics are zero and thus (17) does not hold. Resulting from the application of the Positivstellensatz are the SOS matrix multipliers $s_{1}(\bar{x})$ and $s_{2}(\bar{x})$ which certify the constraints, and $l=\epsilon \bar{x}^{T} \bar{x}$ which enforces strict positivity of $C(\bar{x})$. The polynomial degree of the SOS multiplier and $C$ are in theory unlimited and subject to the choice of the user.

The program (20) has bilinear terms in the coefficients of $s_{1}$ and $\rho / Z$. A local optimum of (20) can be obtained by turning the bilinear problem into a series of convex problems. This is done by iteratively fixing $\rho$ and $Z$ while solving (20) to obtain feasible SOS multipliers, and then solving (20) by maximizing $\rho$ over $Z$ while keeping the obtained multipliers fixed, until the result for $\rho$ converges. Each of the iterative steps is linear in the polynomial coefficients and can thus be solved as a semi-definite program (SDP). This procedure can be initialized with a unit matrix $Z$ and a bisection on $\rho$.

Remark 1: Due to the fact that conditions (20b), (20c) and (20d) represent SOS matrix constraints, solving the program (20) implies an SOS program with $2 n(p+1)$ indeterminants. Due to the current capability of standard SDP solvers the method is not yet applicable to problems with more than 4 to 6 PCE states, depending on the polynomial degree of the problem. Current progress towards more powerful methods to solve SOS programs, such as DSOS and SDSOS programs [27], could offer a remedy.

\section{B. Step 2: Finding an invariant region}

In Step 2, the ROTC inner estimate $\overline{\mathcal{R}}$ is obtained as the largest invariant region inside the contracting region $\overline{\mathcal{Z}}$ obtained in Step 1. In order to find the invariant set $\overline{\mathcal{R}}$, condition (18) can be directly tested on the boundary of $\overline{\mathcal{Z}}$. In general, for a polynomial function $r(\bar{x})=v(\bar{x})^{T} R v(\bar{x})$ an invariant region $\overline{\mathcal{R}}=\left\{\bar{x} \mid r(\bar{x}) \leq \rho_{\text {inv }}\right\} \subseteq \overline{\mathcal{Z}}$ can be obtained by applying the Positivstellensatz as in Step 1 to the set containment condition on the invariant set. Setting $\partial(z)=\partial(r)$ and $\rho_{\text {inv }}=\rho$, where $\rho$ is obtained in Step 1, the following SOS optimization program is formulated. The program is started with the largest possible set inside $\overline{\mathcal{Z}}$, 
e.g., the set $\overline{\mathcal{Z}}$ itself, which is then adjusted in the direction of decreasing derivatives at the boundary of the set until condition (18) is satisfied.

$$
\begin{aligned}
& \text { find } R, h(\bar{x}), s(\bar{x}), \nu \\
& \text { subject to } \begin{aligned}
& \nu<0 \\
&-\left(\nabla r(\bar{x}) \bar{f}(\bar{x})-\nu \bar{x}^{T} \bar{x}\right)-h(\bar{x})(\rho-r(\bar{x})) \in \Sigma[\bar{x}] \\
&-(\rho-r(\bar{x})) s(\bar{x})-(z(\bar{x})-\rho)) \in \Sigma[\bar{x}] \\
& s(\bar{x}) \in \Sigma[\bar{x}]
\end{aligned}
\end{aligned}
$$

The SOS polynomial $s(\bar{x})$ certifies the set containment $\overline{\mathcal{R}} \subseteq \overline{\mathcal{Z}}$ in (21d), and the indefinite polynomial multiplier $h(\bar{x})$ results from the equality constraint in (18) imposed in (21c). The variable $\nu$ is used to find an invariant region $\overline{\mathcal{R}}$. Since this invariant region is characterized by inwards pointing dynamics at the boundary, an invariant region is found as soon as a negative value for $\nu$ has been reached. Program (21) is thus initialized with a feasible positive $\nu$ and (21) is repeatedly solved until a negative $\nu$ is found. Due to bilinear terms in the decision variables, program (21) is solved in the same iterative fashion as program (20).

\section{Algorithmic options for the computation of $\overline{\mathcal{R}}$}

If program (21) cannot find a region for which $\nu$ is negative the reasons can be the following: Either, the contracting region $\overline{\mathcal{Z}}$ obtained in Step 1 is too small to contain an invariant region, or the invariant region inside $\overline{\mathcal{Z}}$ has a shape which cannot be fitted with a sublevel set of the polynomial function $r$. In the first case, a larger region can be searched for by increasing the polynomial degree of the metric and/or SOS multipliers in Step 1. In the second case, the polynomial degree of both $z$ and $r$ can be increased. To allow for both Step 1 and Step 2 to result in larger ROTC estimates, more flexible metrics and regions can be considered. For example, composite metrics and regions (similar to an approach taken for composite Lyapunov functions in [25]) or locally piecewise constructed metrics (similar to an approach for piecewise affine metrics in [9]) can be employed.

\section{ILLUSTRATIVE EXAMPLE}

We demonstrate the method on a stochastic system previously considered in a deterministic version in [9],

$$
\begin{aligned}
& \dot{x}_{1}=-x_{1}+x_{2}, \\
& \dot{x}_{2}=x_{1}-(2+\phi(\xi)) x_{2} .
\end{aligned}
$$

The stochastic parameter $\phi$ comes from a Gaussian distribution with mean $m=0$ and variance $\sigma^{2}=0.2$, i.e. the random variable $\xi$ has standard normal distribution. As a basis for the PC expansion the Hermite polynomials are chosen as these give optimal convergence in the $\mathcal{L}_{2}$-sense for normal distributions. A truncation order $p=2$ was used to capture the significant PCE modes, the higher order modes were negligibly small. For a metric inverse with $\partial(C)=2$, SOS multiplier degrees $\partial\left(s_{1}\right)=2, \partial\left(s_{2}\right)=0, \partial(\lambda)=$ $0, \partial(s)=2, \partial(h)=2$, a quadratic invariant and contracting region with $R$ being the identity matrix $\mathbb{I}^{6 \times 6}$ was found with a radius $\rho=5$.

\section{REFERENCES}

[1] I. R. Manchester, M. M. Tobenkin, M. Levashov, and R. Tedrake, "Regions of Attraction for Hybrid Limit Cycles of Walking Robots," in IFAC Proc. Vol. IFAC, 2011, pp. 5801-5806.

[2] X. Chen and C. Fengde, "Stable periodic solution of a discrete periodic Lotka-Volterra competition system with a feedback control," Appl. Math. Comput., vol. 181, no. 2, pp. 1446-1454, 2006.

[3] D. R. Millman, I. P. King, and P. S. Beran, "A stochastic approach for predicting bifurcation of a pitch and plunge airfoil," 21st AIAA Appl. Aerodyn. Conf., no. June, 2003.

[4] E. Ahbe, T. A. Wood, and R. S. Smith, "Transverse contraction-based stability analysis for periodic trajectories of controlled power kites with model uncertainty," in 2018 IEEE Conf. Decis. Control. IEEE, 2018, pp. 6501-6506.

[5] S. Heim and A. Spröwitz, "Beyond Basins of Attraction: Quantifying Robustness of Natural Dynamics," IEEE Trans. Robot., vol. 35, no. 4, pp. 939-952, 2019.

[6] A. Iannelli, A. Marcos, and M. Lowenberg, "Nonlinear Robust Approaches to Study Stability and Postcritical Behavior of an Aeroelastic Plant," IEEE Trans. Control Syst. Technol., vol. 27, no. 2, pp. 703-716, 2019.

[7] W. Lohmiller and J.-J. E. Slotine, "On Contraction Analysis for Nonlinear Systems," Automatica, vol. 34, no. 6, pp. 683-696, 1998.

[8] I. R. Manchester and J.-J. E. Slotine, "Transverse contraction criteria for existence, stability, and robustness of a limit cycle," Syst. Control Lett., vol. 63, pp. 32-38, 2014.

[9] P. Giesl and S. Hafstein, "Construction of a CPA contraction metric for periodic orbits using semidefinite optimization," Nonlinear Anal., vol. 86, pp. 114-134, 2013.

[10] D. Xiu and G. E. M. Karniadakis, "Modeling uncertainty in flow simulations via generalized polynomial chaos," J. Comput. Phys., vol. 187, no. 1, pp. 137-167, 2003.

[11] O. Le Maitre and O. Knio, Spectral methods for uncertainty quantification. Springer, 2010.

[12] E. Ahbe, A. Iannelli, and R. S. Smith, "Region of attraction analysis of nonlinear stochastic systems using Polynomial Chaos Expansion," ArXiv, p. 1911.00252v1, 2019.

[13] C. L. Pettit and P. S. Beran, "Polynomial chaos expansion applied to airfoil limit cycle oscillations," Collect. Tech. Pap. AIAA/ASME/ASCE/AHS/ASC Struct. Struct. Dyn. Mater. Conf., vol. 3, no. April, pp. 1975-1985, 2004.

[14] P. S. Beran, C. L. Pettit, and D. R. Millman, "Uncertainty quantification of limit-cycle oscillations," J. Comput. Phys., vol. 217, no. 1, pp. 217-247, 2006.

[15] E. M. Aylward, P. A. Parrilo, and J.-J. E. Slotine, "Stability and robustness analysis of nonlinear systems via contraction metrics and SOS programming," Automatica, vol. 44, pp. 2163-2170, 2008.

[16] F. S. Hover and M. S. Triantafyllou, "Application of polynomial chaos in stability and control," Automatica, vol. 42, no. 5, pp. 789-795, 2006.

[17] J. K. Hale, Ordinary Differential Equations. R.E. Krierger Pub. Co., New York, 1980.

[18] C. L. Pettit and P. S. Beran, "Spectral and multiresolution Wiener expansions of oscillatory stochastic processes," J. Sound Vib., vol. 294, no. 4, pp. 752-779, 2006.

[19] D. Xiu, I. G. Kevrekidis, and R. Ghanem, "An equation-free, multiscale approach to uncertainty quantification," Multiphysics Model., vol. May/June, pp. 16-23, 2005.

[20] L. Fagiano, M. Khammash, and C. Novara, "On the guaranteed accuracy of Polynomial Chaos Expansions," in Proc. IEEE Conf. Decis. Control. IEEE, 2011, pp. 728-733.

[21] T. Mühlpfordt, R. Findeisen, V. Hagenmeyer, and T. Faulwasser, "Comments on truncation errors for Polynomial Chaos Expansions," IEEE Control Syst. Lett., vol. 2, no. 1, pp. 169-174, 2018.

[22] G. Valmorbida and J. Anderson, "Region of attraction estimation using invariant sets and rational Lyapunov functions," Automatica, vol. 75, pp. 37-45, 2017.

[23] H. Khalil, Nonlinear systems, 3rd ed. Prentice Hall, Upper Saddle River, 2002.

[24] P. A. Parrilo, "Structured semidefinite programs and semialgebraic geometry methods in robustness and optimization," Ph.D. dissertation, California Institute of Technology, 2000.

[25] W. Tan and A. Packard, "Stability Region Analysis Using Polynomial and Composite Polynomial Lyapunov Functions and Sum-of-Squares Programming," IEEE Trans. Automat. Contr., vol. 53, no. 2, pp. 565571, 2008. 
[26] U. Topcu, A. K. Packard, P. Seiler, and G. J. Balas, "Robust regionof-attraction estimation," IEEE Trans. Automat. Contr., vol. 55, no. 1, pp. 137-142, 2010.

[27] A. A. Ahmadi and A. Majumdar, "DSOS and SDSOS optimization: More tractable alternatives to sum of squares and semidefinite optimization," SIAM J. Appl. Algebr. Geom., vol. 3, no. 2, pp. 193-230, 2019. 